Mittlere Örter der Vergleichsterne.

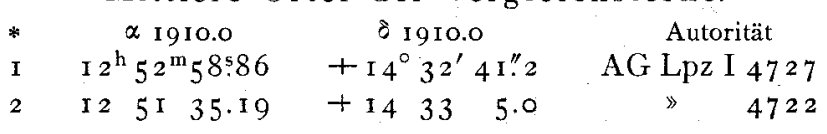

März I 5 . Vergr. $=250$, günstige Bedingungen für die Messung. - März I6. Distanz sehr weit, deswegen nur Vergr. $=90$. Der Planet erscheint auffallend hell gegen gestern. - März 2 I. Bei etwas verschleiertem Himmel und Mondschein wegen Lichtschwäche nicht $z u$ messen. Die Korrektionen der Ephemeride (A. N. 4392) sind März I5 $+0.26+1.5$ sowie März $16+0.25$ - I". 9 .

Ähnliche Wahrnehmungen bezüglich der Helligkeit des Planeten wie die vorstehend mitgeteilten hatte ich auch schon zur Zeit der Opposition 1906 gemacht. Da die Möglichkeit nicht von der Hand zu weisen war, daß dieser in photometrischer Hinsicht wenig untersuchte Planet vielleicht eine periodische Lichtschwankung erleide, so wurde damals eine Reihe von Helligkeitsbestimmungen nach der Argelanderschen Methode ausgeführt. Das dazu benutzte Instrument war der Sechszöller der Berliner Urania, bei dem meist 6o-fache Vergrößerung angewendet wurde. Die Bestimmung der Helligkeit der Egeria erfolgte im Anschluß an die Angaben der BD und zwar in der Weise, daß deren Daten soweit korrigiert wurden, daß sie unseren Stufenschätzungen entsprachen, während die Summe der angebrachten Korrektionen zu null gemacht wurde. Es wurden meist mehrere Gruppen von Schätzungen gemacht, deren Ergebnisse jedoch nie mehr als $o^{\mathrm{m}} \mathrm{I}$ voneinander abwichen. Deswegen sind sie in der

Berlin, I9 I I März I 7 . folgenden Übersicht einfach in Mittelwerte zusammengezogen. Reduziert man die Schätzungsergebnisse mit dem mittleren Phasenkoeffizienten der kleinen Planeten, der nach Müller Photom. d. Gest. p. 378 rund $z u \quad 0.03$ angenommen wurde, so erhält man für $m_{0}$ den Wert $9^{\mathrm{m}} \cdot 4 \mathrm{I}$ statt $9^{\mathrm{m}} \cdot 7$, wie sonst angenommen wird (V.R.I. 16 p. 28 ). Luft $5=$ sehr klar.

\begin{tabular}{|c|c|c|c|c|}
\hline I 906 & M. E. Z. & Luft & Größe & $B-R$ \\
\hline April 5 & I I ${ }^{h} \cdot 9^{-I} 3^{h} \cdot 2$ & $2-3$ & $9^{m} \cdot 43$ & $0 \% 00$ \\
\hline IC & I $1.7-12.4$ & 2 & $9 \cdot 39$ & 0.0 \\
\hline I 6 & $9 \cdot 5$ & 2 & 9.42 & -0.02 \\
\hline 23 & $12.0-\mathrm{I} 3 . \mathrm{I}$ & $3-4$ & 9.57 & +0.03 \\
\hline Mai & I 2.6 & $3^{-2}$ & 9.73 & 0.00 \\
\hline
\end{tabular}

-April 5. Mond stört etwas. Farbe blaß orangerötlich. - April 23. Merklich rötlichorange.

In dem Gang der $B-R$ ist keine Veränderung der mittleren Helligkeit der Egeria ausgesprochen. Die ig io März 2 I bemerkte Lichtschwäche hängt wahrscheinlich mit der Färbung der kleinen Planeten überhaupt zusammen, die im Durchschnitt gelblicher bezw. rötlicher als die Fixsterne sind. Es findet damit die Wahrnehmung von Herrn Dr. W. Luther (A. N. Bd. I87.346) eine Bestätigung, »daß das Planetenlicht in stärkerem Maße durch einsetzende geringere Durchsichtigkeit der Luft geschwächt werde als das Fixsternlicht «.

H. H. Kritzinger.

Observations de la Comète de Halley $1909 \mathrm{c}$

faites à l'Observatoire de Besançon avec l'Equatorial droit de 2 I cm par Paul Brick.

\begin{tabular}{|c|c|c|c|c|c|c|c|c|c|c|c|c|c|c|c|c|}
\hline \multicolumn{2}{|c|}{1910} & T.m.Besanç. & $\lambda \alpha$ & \multicolumn{2}{|r|}{$A \delta$} & Cp. & \multicolumn{2}{|r|}{$\alpha$ app. } & $\log p \cdot A$ & \multicolumn{3}{|c|}{$\delta$ app. } & $\log p \cdot A$ & \multicolumn{2}{|c|}{ Red.ad 1. app. } & \multirow{2}{*}{$\begin{array}{r}4 \\
\text { I }\end{array}$} \\
\hline 'évı & I & $7^{\mathrm{h}} 4 \mathrm{I}^{\mathrm{m}} 7^{\mathrm{s}}$ & $+o^{m} 51: 30$ & & -- & I $2,-$ & & $2^{\mathrm{m}} 50^{\mathrm{s}} \cdot 34$ & 9.496 & & - & & - & $-\mathrm{I}_{1}^{\mathrm{s}_{\mathrm{I}} 8}$ & & \\
\hline Avril & 27 & I $5 \quad 46 \quad 44$ & +0.23 .20 & + & $2^{\prime} \quad 7.9$ & IO, IO & 23 & $5046.1 \mathrm{I}$ & $9.607 \mathrm{n}$ & + & $7^{\circ} 55^{\prime}$ & I 8.6 & 0.803 & $-\mathbf{I} .00$ & -8.3 & 2 \\
\hline \multirow[t]{7}{*}{ Mai } & I 3 & I $5 \quad 3^{8} \quad 37$ & +442.62 & - & 914.6 & I 2,9 & $\circ$ & $\begin{array}{ll}57 \quad 5^{2.77}\end{array}$ & 9.6 I $5 n$ & +1 & 3 & 13.0 & 0.796 & -0.92 & $-5 \cdot 7$ & 3 \\
\hline & 27 & $946 \quad 6$ & - I 4.67 & & - & $3,-$ & 9 & $175 \mathrm{I} .55$ & 9.582 & & - & & - & -0.08 & 0.0 & 4 \\
\hline & 27 & $9 \quad 5^{I} \quad$ I 5 & - & -1 & 431.2 &,- 3 & & & - & + & $52 \mathrm{I}$ & 48.6 & 0.797 & -0.08 & 0.0 & 4 \\
\hline & 28 & $\begin{array}{lll}9 & 22 & 33\end{array}$ & -. $0 \quad 4.27$ & & - & $6,--$ & & $28 \quad 18.75$ & 9.559 & & - & & - & -0.04 & -0.5 & 5 \\
\hline & 28 & $926 \quad 19$ & - & - & 2.9 &,- 4 & & - & - & + & $4 \quad 27$ & 17.2 & 0.795 & -0.04 & -0.5 & 5 \\
\hline & $3 r$ & 9443 & $-2 \quad 21.24$ & & - & $6,-$ & & 5026.56 & $9 \cdot 5^{67}$ & & - & & -- & +0.05 & -1.5 & 6 \\
\hline & $3 \mathrm{I}$ & $9 \quad 52 \quad 18$ & - & - & 230.6 &,- 2 & & - & - & + & 229 & $35 \cdot 7$ & 0.803 & +0.05 & $-I \cdot 5$ & 6 \\
\hline \multirow[t]{4}{*}{ Juin } & 7 & $\begin{array}{lll}8 & 56 & 3\end{array}$ & +o 59.99 & - & 2.7 & 3,6 & 10 & 2.43 & $9 \cdot 521$ & + & 0 I 1 & 27.2 & $0.8 \mathrm{I} \mathrm{I}$ & +o.10 & -2.3 & 7 \\
\hline & 7 & $\begin{array}{lll}8 & 56 & 31\end{array}$ & -o 43.48 & $\cdots$ & $2 \quad 25.9$ & 3,3 & IO & 2.57 & $9 \cdot 52 \mathrm{I}$ & + & o 1 I & 29.2 & $0.8 \mathrm{I} \mathbf{I}$ & +0.11 & -2.4 & 8 \\
\hline & 8 & $9 \quad 5053$ & -I 48.19 & - & 444.0 & 6,3 & IO & I $826.9 \mathrm{I}$ & 9.574 & - & $\circ \quad I$ & 27.0 & 0.8 I I & +0.12 & -2.4 & 9 \\
\hline & 19 & $9 \quad 54 \quad 37$ & to 41.98 & + & 946.6 & $5, \mathbf{I}$ & IO & $3^{8} \quad 55.5 \mathrm{I}$ & $9.59 \mathrm{~F}$ & - & I 32 & 35.6 & $0.8 \mathrm{r} 3$ & +0.11 & -2.7 & Io \\
\hline
\end{tabular}

Etoiles de comparaison.

\begin{tabular}{|c|c|c|c|}
\hline$*$ & $\alpha$ I9 I0.0 & $\delta 1910.0$ & Autorité \\
\hline I & $1^{\mathrm{h}} \quad 2^{\mathrm{m}} \quad 0.22$ & $+8^{\circ} 23^{\prime} \quad 9^{\prime \prime} 4$ & $\mathrm{AGLpz} \mathrm{II} 40 \mathrm{I}$ \\
\hline 2 & $23 \quad 5023.9 \mathrm{I}$ & +75319.0 & I I 8 I 3 \\
\hline 3 & $\circ 53$ I I.07 & +131233.3 & AG Lpz I $25^{6}$ \\
\hline 4 & 9 I $8 \quad 5^{6.30}$ & +536 19.8 & AG Lpz II 5097 \\
\hline 5 & $928 \quad 23.06$ & +43420.6 & AG Alb 3809 \\
\hline
\end{tabular}


Remarques. Le grossissement employé est 57. - Avril 27. Noyau très marqué, commencement de queue. Les trois derniers pointés en $\delta$ ont été faits à l'aide d'un fil d'araignée, le champ étant éclairé par l'aurore. - Mai I 3. Pointés au fil d'araignée. La comète est bien visible à l'œil nu. Condensation centrale très belle, un peu allongée.

Observatoıre de Besançon, i 9 í Mai 4.

Paul Brück.

\section{Beobachtungen des Fayeschen Kometen 1910 e}

am $32.5 \mathrm{~cm}$-Refraktor der Königsberger Sternwarte von $E$. Fost und $K$. Rudolph.

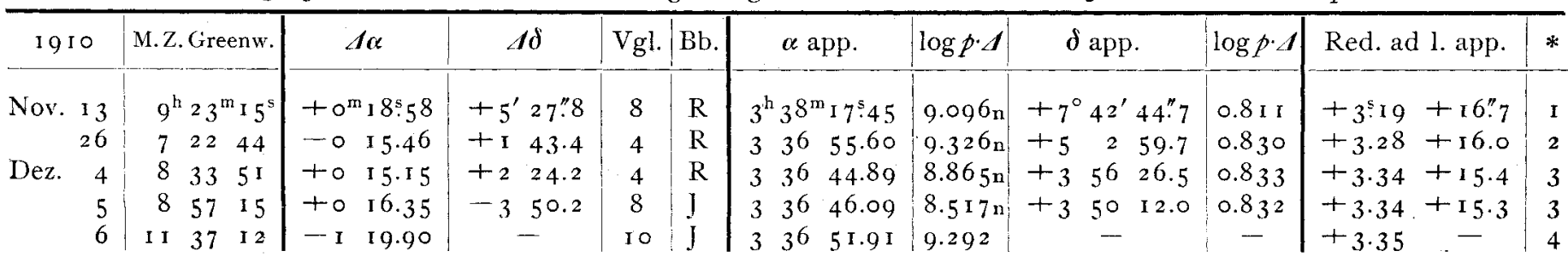

Nov. $\mathrm{r}$ 3. Der Komet erschien als äußerst schwacher verwaschener Nebel ohne Schweif. Einstellung wegen Mondschein und Cirruswolken unsicher. - Dez. 5. Komet im Dunst schwach. - Dez. 6. Komet verschwindet in Wolken.

Mittlere Örter der Vergleichsterne.

\begin{tabular}{|c|c|c|c|c|c|c|c|}
\hline$*$ & $\alpha$ I9I0.0 & $\delta \times 910.0$ & Autorität & $*$ & $\alpha \quad 1910.0$ & $\delta$ I 910.0 & Autorität \\
\hline
\end{tabular}

\section{Osservazioni di alcuni piccoli pianeti}

fatte al R. Osservatorio Astronomico di Milano.

(Equatoriale di $0.218 \mathrm{~m}$ di apertura; distanza focale $3.15 \mathrm{~m}$; micrometro ad anelli; ingrandimento 76 ).

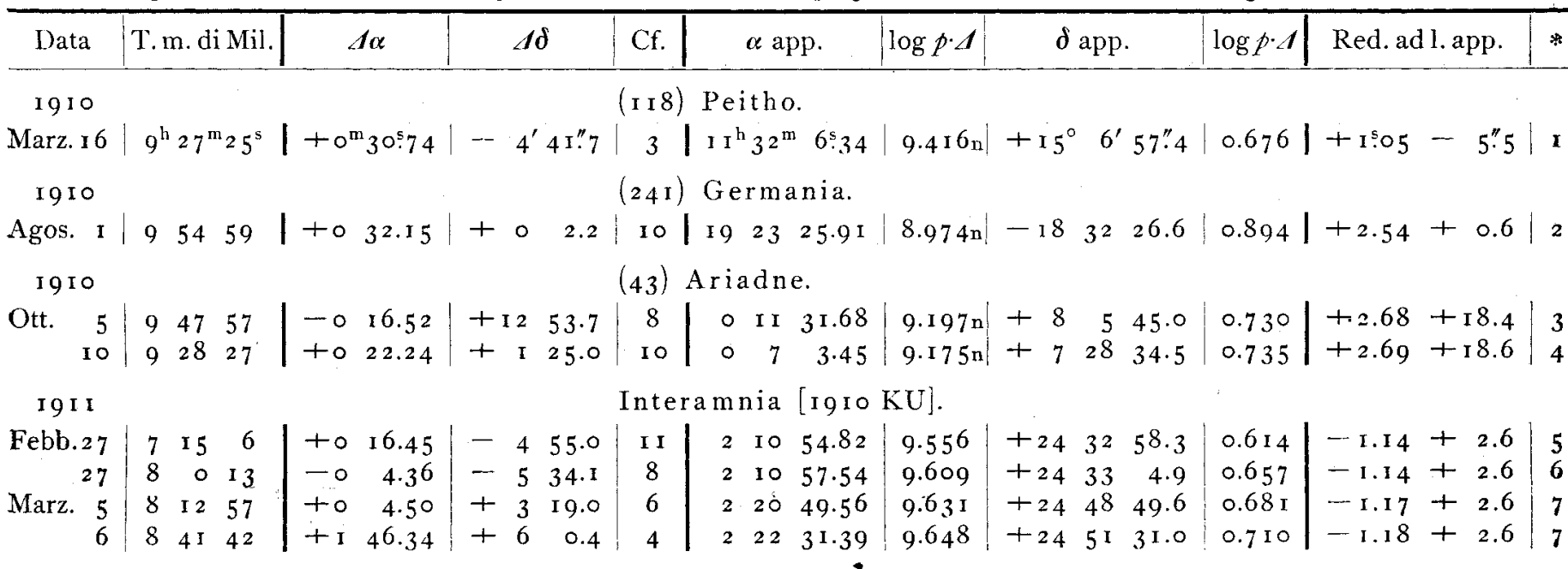

1911

(96) Aegle.

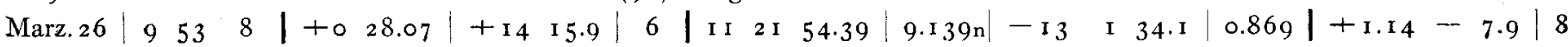

Posizioni medie delle stelle di paragone.

\begin{tabular}{|c|c|c|c|}
\hline$*$ & $\alpha \times 910.0$ & $\delta$ I 910.0 & Autorità \\
\hline $\mathbf{r}$ & $\mathrm{II}^{\mathrm{h}} 3 \mathrm{I}^{\mathrm{m}} 34^{\mathrm{s}} \cdot 55$ & + I $5^{\circ}$ I I' $44^{\prime \prime} 6$ & AG Berl A 4467 \\
\hline 2 & 19.2251 .22 & $\begin{array}{lll}-18 & 32 & 29.4\end{array}$ & $A W_{15398}$ \\
\hline 3 & $\circ$ II 45.52 & +75232.9 & $\mathrm{AG} \mathrm{Lpz} \mathrm{II} 5^{8}$ \\
\hline 4 & $063^{8.5^{2}}$ & +72650.9 & 27 \\
\hline
\end{tabular}

Milano, I9 I I Aprile I 2.

\begin{tabular}{|c|c|c|c|}
\hline$*$ & $\alpha$ I911.0 & $\delta$ I9II.0 & Autorità \\
\hline 5 & $2^{\mathrm{h}} 10^{\mathrm{m}} 395^{\mathrm{s}} \mathrm{I}$ & $+24^{\circ} 37^{\prime} 50^{\prime \prime} .7$ & AG Berl B 68 I \\
\hline 6 & 2 I I 3.04 & $+243^{8} \quad 3^{6.4}$ & 686 \\
\hline $\begin{array}{l}7 \\
8\end{array}$ & $\begin{array}{rrr}2 & 20 & 46.23 \\
\text { I I } & 2 \mathrm{I} & 25.18\end{array}$ & $\begin{array}{lll}+24 & 45 & 28.0 \\
-13 & \text { I } 5 & 42.1\end{array}$ & $\mathrm{RC}_{90}{ }^{\prime \prime} 256^{734}$ \\
\hline
\end{tabular}

L. Gabba. 